\title{
Gamma-Ray Peak Shapes from Cadmium Zinc Telluride Detectors
}

\author{
M. N. Namboodiri \\ A. D. Lavietes \\ J. H. McQuaid
}

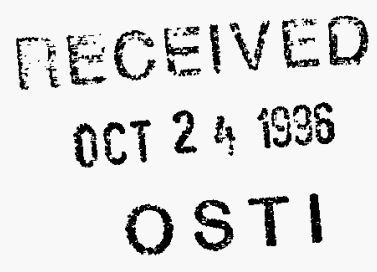

Nuclear Science Applications Section

Isotope Sciences Division

Chemistry \& Materials Science Department

Lawrence Livermore National Laboratory

Livermore, California

September 1996
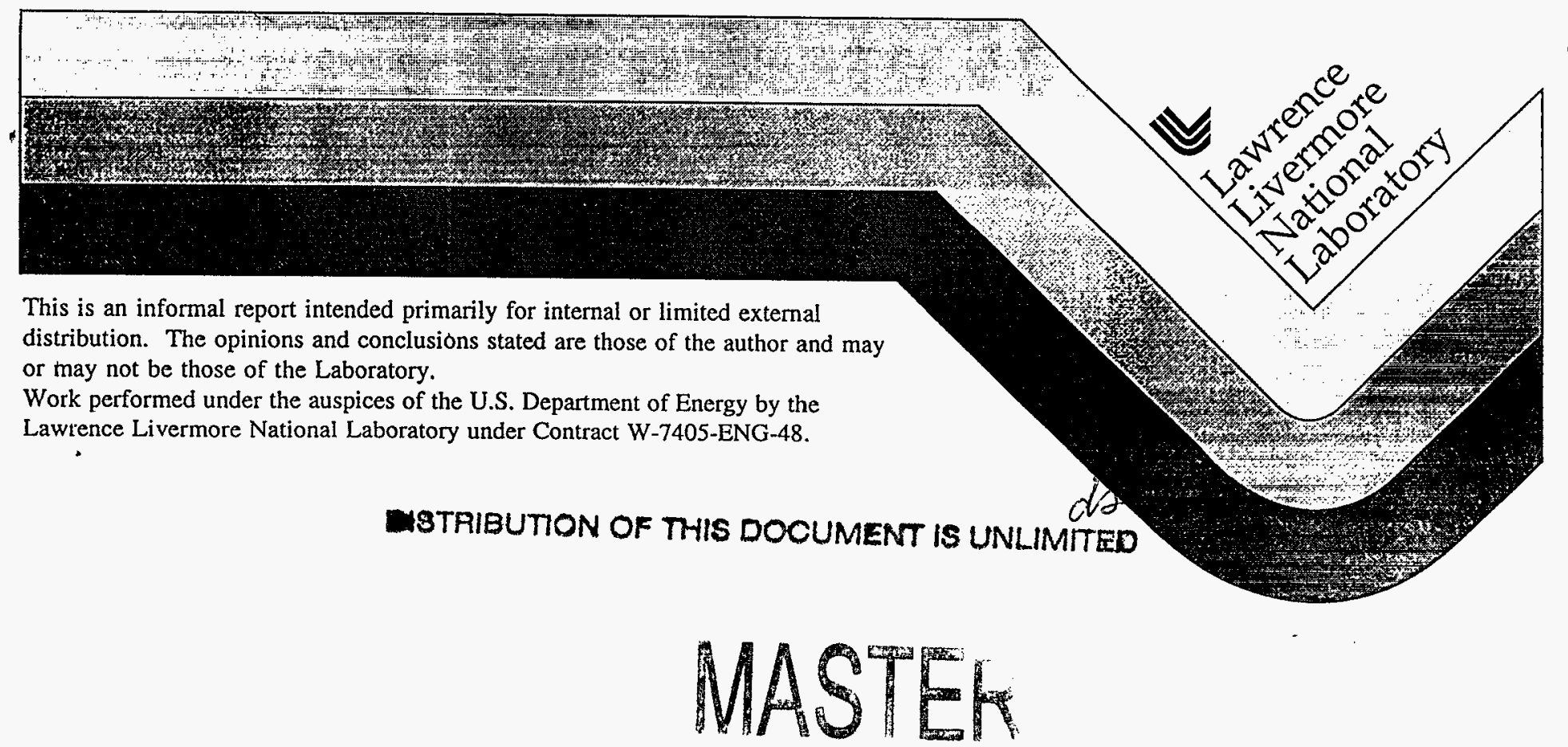


\section{DISCLAIMER}

This documènt was prepared as an account of work sponsored by an agency of the United States Government. Neither the United States Government nor the University of California nor any of their employees, makes any warranty, express or implied, or assumes any legal liability or responsibility for the accuracy, completeness, or usefulness of any information, apparatus, product, or process disclosed, or represents that its use would not infringe privately owned rights. Reference herein to any specific commercial products, process, or service by trade name, trademark, manufacturer, or otherwise, does not necessarily constitute or imply its endorsement, recommendation, or favoring by the United States Government or the University of California. The views and opinions of authors expressed herein do not necessarily state or reflect those of the United States Government or the University of California, and shall not be used for advertising or product endorsement purposes.

This report has been reproduced directly from the best available copy.

Available to DOE and DOE contractors from the Office of Scientific and Technical Information

P.O. Box 62, Oak Ridge, TN 37831

Prices available from (615) 576-8401

Available to the public from the

National Technical Information Service

U.S. Department of Commerce

5285 Port Royal Rd.,

Springfield, VA 22161 


\title{
Gamma-Ray Peak Shapes from Cadmium Zinc Telluride Detectors*
}

\author{
M.N. Namboodiri, A.D. Lavietes and J.H. McQuaid \\ Lawrence Livermore National Laboratory, Livermore, CA 94550
}

\begin{abstract}
We report the results of a study of the peak shapes in the gamma spectra measured using several $5 \times 5 \times 5 \mathrm{~mm}^{3}$ cadmium zinc telluride (CZT) detectors. A simple parameterization involving a Gaussian and an exponential low energy tail describes the peak shapes well. We present the variation of the parameters with gamma energy. This type of information is very useful in the analysis of complex gamma spectra consisting of many peaks.
\end{abstract}

\section{Introduction}

Gamma-ray detectors made of cadmium telluride ( $\mathrm{CdTe}$ ) and cadmium zinc telluride (CZT) are finding numerous applications in fields such as the assay and isotopic analysis of nuclear materials 1,2 . They have a comparatively high detection efficiency for gamma rays because of the high atomic numbers of their constituents and they can be operated at room temperatures. Of these two materials, CZT is superior because of its higher resistivity and higher band gap (1.6 ev for CZT compared to $1.47 \mathrm{ev}$ for CdTe). Reasonable size detectors with good enough resolution for many applications are now being fabricated from CZT material made by a modified high pressure Bridgeman crystal growth technique 3,4 . Portable instruments incorporating such detectors are being developed 5 at our Laboratory.

Gamma spectra obtained with CdTe and CZT detectors usually have a significant tail on the low energy side of the full energy peak. This arises from the unfavorable charge transport properties of the detector material itself. Thus, holes have low mean free paths in the material and are trapped with high probability. In CdTe, for example, the mobility-life time product, $\mu \tau$ is about $6 \mathrm{x}$ $10^{-4}$ for electrons and $3 \times 10^{-5}$ for holes 6 . Thus, at a field of $1000 \mathrm{~V} / \mathrm{cm}$, the mean free path of holes is only a fraction of a $\mathrm{mm}$.

Detector resolution can be improved by discarding pulses on the basis of rise time, but this reduces the detector efficiency greatly. Several methods have been proposed for correcting for the effect of the incomplete charge collection. Some of these involve the use of the rise time of each pulse in addition to the pulse height 7,8 . Recently, a neural network algorithm has been used for enhancing the energy resolution of gamma spectra taken with a CdTe detector 9 . With the availability of relatively large volume (e.g. $5 \times 5 \times 5 \mathrm{~mm}^{3}$ ) CZT detectors with reasonably good resolution, special correction methods for the low energy tailing are not essential for a variety of applications.

Nevertheless, one must understand the characteristic shape of the peaks in the gamma spectra (including the amplitude and shape of the tail) in order to analyze complex gamma spectra obtained using these detectors. For this 


\section{DISCLAIMER}

Portions of this document may be illegible in electronic image products. Images are produced from the best available original document. 
reason, we have studied the peak shapes of gamma rays as a function of energy for CZT detectors.

\section{Experimental Details}

In this work, we have mainly used CZT detectors made by eV Products using the high pressure Bridgeman technique ${ }^{3}$. These detectors contain 10 atom per cent $\mathrm{Zn}$, thus having the composition Cdo.9Zno.1Te. Most of the work described in this report was done with a $5 \times 5 \times 5 \mathrm{~mm}^{3}$ detector (designated here as detector A) which had a resolution of $2.7 \mathrm{keV}$ (FWHM) for the $122 \mathrm{keV}$ gamma ray from ${ }^{57} \mathrm{Co}$. In this work, we used the special electronics developed for the portable CZT system 5 , in which the preamplifier and filter amplifier have been designed to minimize the effects of poor hole-mobility and charge trapping (which give rise to the tail in the spectrum) and of noise (primarily shot noise).

The electronic contribution (pulser resolution without the detector) is 1.2 keV FWHM using $0.5 \mu$ s shaping ( $1.0 \mu$ s peaking). The electronic system is shown in Fig. 1. This monolithic design was able to achieve good resolution ( $10 \%$ better than commercial instrumentation) and low power consumption (600 $\mathrm{mW}$ ). A low noise field-effect transistor (FET) was selected for optimization of series and parallel noise, front end gain, and rise time. Good stability is achieved by running a $400 \mathrm{MHz}$ opamp (IC1) in an open loop configuration with DC feedback for stability. This IC is a critical component that achieves the necessary combination of high bandwidth and low noise. This portion of the preamplifier is usually a hybrid circuit, which is physically larger and has higher power consumption. Power consumption is reduced in the present design by running IC1 on a single ended supply $(+12 \mathrm{~V})$. The preamplifier has a sensitivity of $20 \mathrm{~V} / \mathrm{pC}$ and a rise time of $24 \mathrm{~ns}$.

The amplifier stage is voltage-sensitive and is composed of a low noise, high bandwidth opamp preceded by a differentiator and pole-zero compensation network. A two-stage Sallen-Key filter was designed using opamps IC3 and IC4. This circuit has two pairs of complex poles and was found to be very stable. This circuit also has a sharp noise-corner response, which is very important in reducing parallel noise and at the same time minimizing ballistic deficit due to the slow charge collection of the detector. The shaping time of this filter is $0.5 \mu \mathrm{s}$ ( $1.0 \mu$ s peaking time). The optimum shaping time for the electronics is 4-6 $\mu \mathrm{s}$, based on the capacitance of the detector and other circuit parameters. However, charge trapping in the detector has a pronounced effect on resolution, and as a result, the filter time constant must be reduced to $0.5 \mu \mathrm{s}$. This reduces the amount of low-energy tailing. Measurements using several CZT detectors have shown that trapping can add 2-3 keV (in quadrature) to the resolution if the filter time constant is increased to $1 \mu \mathrm{s}$.

The second stage of the filter was designed with a higher $Q$ than the first stage This design achieves improved performance and results in a nearly Gaussian output pulse shape.

Results and Discussion 
We studied the peak shapes in gamma spectra taken with these detectors using standard gamma ray sources. We used gamma rays from $57 \mathrm{Co}$, ${ }^{133} \mathrm{Ba},{ }^{137} \mathrm{Cs},{ }^{152} \mathrm{Eu}$ and ${ }^{241} \mathrm{Am}$, sources, thus covering the energy range 59 to $661 \mathrm{keV}$.

Fig. 2 a shows a spectrum 57 Co taken with this detector. The characteristic low energy tailing is very evident in this spectrum. This may be compared to the spectrum shown in Fig 2(b) which was calculated using the Monte Carlo transport code MCNP10. This calculation gives an ideal spectrum which does not include the effect of dispersion in the number of charge carrier pairs produced when the gamma ray interacts within the detector, the trapping of carriers and the finite resolution of the electronics. The calculated spectrum shows small peaks in the $80-90$ and $105-115 \mathrm{keV}$ regions which correspond to the escape of $\mathrm{Cd}, \mathrm{Zn}$ and Te $\mathrm{X}$-rays generated in the interaction of the 122 and $136.5 \mathrm{keV}$ gamma rays in the detector. These peaks also appear as a smeared out bump in the experimental spectrum. The MCNP spectrum also shows the Compton edges at 39.4 and $46.4 \mathrm{keV}$ corresponding to the two the 122 and $136.5 \mathrm{keV}$ gammas. These do not stand out over the background in the experimental spectrum. The experimental spectrum also has the $14.4 \mathrm{keV}$ gamma which occurs in a fraction of the decay of ${ }^{57} \mathrm{Co}$. It was not included in the MCNP calculation.

\section{Peak shapes:}

The low energy tails in gamma spectra have been analyzed often in terms of two components, i.e. a long term and a short term tail ${ }^{11}$. In the present work, we have treated the part of the tail extending over tens of $\mathrm{keV}$ as background and concentrated on the short range part of the tail. After subtracting the background (as calculated by the method given in Ref. 11), the peaks were fit to a sum of a Gaussian and an exponential tail confined to the low energy side of the Gaussian. This description of the line shape is the same as that used in earlier work on Ge detectors at LLNL11.

In the fits, there are five parameters in addition to the overall normalization: the peak energy and the width of the Gaussian, the relative amplitude and the slope of the exponential tail, and a cutoff parameter that makes the tail go smoothly to zero at the peak. Specifically, the expression used is:

$$
N_{i}=N_{0}\left\{\exp \left[\alpha\left(\varepsilon_{i}-\varepsilon_{0}\right)^{2}\right]+T\left(\varepsilon_{i}\right)\right\}
$$

where

$$
\begin{aligned}
& N_{i}=\text { net counts in channel } \varepsilon_{i} \\
& N_{0}=\text { net counts at the peak, } \\
& \alpha=-1 /\left(2 \sigma^{2}\right)=-2.7726 /(\text { FWHM })^{2} \text { where }
\end{aligned}
$$

FWHM is the full width of the peak at half maximum and $\sigma^{2}$ is the variance of the Gaussian component, 


$$
\begin{array}{lll}
\varepsilon_{0} & = & \text { the mean of the Gaussian, and } \\
T\left(\varepsilon_{i}\right)= & \text { the tail function. }
\end{array}
$$

The tail function is given by

$$
\begin{aligned}
& T\left(\varepsilon_{i}\right)=\left\{A \exp \left[B\left(\varepsilon_{i}-\varepsilon_{0}\right)\right]\right\}\left\{1-\exp \left[C \alpha\left(\varepsilon_{i}-\varepsilon_{0}\right)^{2}\right]\right\} \delta \\
& \text { where } \quad \begin{array}{l}
A=\text { the amplitude of the tail } \\
B=\text { the slope of the tail } \\
C=\text { the tail cut off parameter } \\
\text { and } \quad \delta=1 \text { for } \varepsilon_{i}<\varepsilon_{0} \\
\quad 0 \text { for } \varepsilon_{i}>\varepsilon_{0} .
\end{array}
\end{aligned}
$$

Fig. 3 shows the results of fitting the $122 \mathrm{keV}$ peak in the 57 Co spectrum according to this procedure.

By fitting the intense gamma rays from the other sources mentioned above, the energy dependence of the parameters can be studied. The energy dependence of the various parameters that describe the peak shapes is shown in Figs 4 and 5 . The variance of the Gaussian increases linearly with increasing gamma energy, as shown in Fig 4. Both the slope and the intercept of the line describing this variation are larger than those for a typical Ge detector. Fig. 4 shows the results for our best detector (detector A) and two other detectors (designated $B$ and $C$ ) which have significantly poorer overall resolution. Detectors $B$ and $C$ were from a batch of detectors which were being evaluated for the portable CZT system. The results for the two latter detectors are very similar. We note that the width of their Gaussian components is larger and increases more rapidly than that of detector $A$.

The parameters which describe the tail are shown in Fig. 5. The slope parameters are shown on the left and the tail amplitudes are shown on the right. In both cases, parameters for detector A, are at the bottom and those for detectors $B$ and $C$ are on top. All three detectors exhibit very similar behavior. The slope of the exponential tail varies strongly with energy at low gamma energy, and then becomes nearly constant. The slope is nearly constant with energy for Ge usually. A typical value for Ge detectors is shown in Fig. 5 . The tail amplitude for the CZT is significantly larger than for Ge. The tail cutoff parameter, $C$ is not a very sensitive one in the description of the line shape; it is found to be larger for CZT than for Ge. A constant value of 0.7 was used here at all energies (cf. 0.4 used for Ge in the LLNL codes ${ }^{11}$ ). The comparison with $\mathrm{Ge}$ shown in Figs 4 and 5 highlights the fact that in CZT spectra the low energy tail is much more significant - flatter and higher in amplitude - than in Ge spectra.

We note that the short tail parameters for detectors $B$ and $C$ are very comparable quantitatively to those for detector $A$. This is underscored by the curves shown in Fig 5. The energy dependence of the tail slope and amplitude parameters for detector A (bottom halves of the two panels in Fig 5) was described by the simple expressions shown in the figure caption. The resulting curves for detector A were scaled without change of shape and plotted along with the data in the top halves. It is seen that with a simple change in normalization, the same curves describe the variation of the slope of the tail for all three detectors. The tail amplitudes behave the same way. 
As mentioned already, the low energy tail results mainly from poor charge collection due to charge carrier trapping. This phenomenon will be sensitive to the properties of the material and the details of detector fabrication in so far as they affect charge transport. We may, therefore, expect the nature and extent of tailing to differ in detectors from different manufacturers. As an example, Fig. 6 shows the gamma spectra of ${ }^{57}$ Co taken with a $5 \times 5 \times 5 \mathrm{~mm}^{3}$ CZT detector made by eV Products and a $3 \times 3 \times 3 \mathrm{~mm}^{3}$ detector made by Digirad and. The long-term tail is very much reduced in the spectrum taken with the Digirad detector. The specific differences in the detector material or fabrication technique that brought about this marked improvement are not known to us at this point. Fig. 7 shows a comparison of the fits of the 122-keV peaks in these spectra. Of the two detectors, the Digirad product has the poorer resolution, as shown by the larger width of the Gaussian part of the peak. The very long term tail in the eV Products detector spectrum is subtracted as part of the background in the fitting procedure, as described above. In the Digirad detector spectrum, the tailing is essentially all short-term, and this is not removed significantly in the background subtraction. Therefore, in the present description of the peaks in terms of a Gaussian plus one short-term tail, the Digirad detector spectrum exhibits a tail which is both flatter and has a higher amplitude in relation to the peak of the Gaussian. The Digirad detector spectrum, in spite of the improved peak-to-valley ratio, is more asymmetric at half maximum and above than the eV Products detector spectrum.

\section{Application to Uranium Analysis}

Because of their ambient temperature operation, CZT detectors are very suitable for several applications in the analysis of special nuclear materials in the field of safeguards technology. Software packages are now being developed at LLNL for the analysis of CZT spectra of uranium which use the peak shape description discussed in this report along with the spectral analysis methodology that has been developed here over the years ${ }^{12}$. The energy region from 86 to $102 \mathrm{keV}$ in the uranium spectrum, consisting of thirteen gamma-ray and $X$-ray peaks, is used for this analysis. When analyzing such a complex spectrum covering a range of energy with several peaks of very different intensity, one may have better success if one extends the peak description to include a short and a long term tail and even the $\mathrm{Cd}, \mathrm{Zn}$ and $\mathrm{Te}$ $\mathrm{X}$-ray escape peaks. ${ }^{13 .}$

\section{Trapping-detrapping Model}

As pointed out in the introduction, several approaches have been used for correcting the energy spectra for the tailing. Even though in our application of CZT detectors we are relying, instead, on the knowledge of peak shapes for unraveling complex gamma spectra2,12, it is still of interest to understand the processes that lead to the tailing. Reports in the literature describe studies which attempt to model the effect of carrier trapping and detrapping on the shape of the final observed gamma spectrum8,14 A Monte Carlo code can be used to calculate the energy deposited by gamma ray interactions in different layers of the detector. One can then calculate the contribution to the final 
detected pulse height from the charge carriers produced at different depths, taking into account the effect of trapping and detrapping as the carriers drift to the collecting electrodes. It has been shown that the prominent tails observed in CdTe spectra can be understood on the basis of such a model. We are exploring such a model to understand the energy variation of peak shape parameters for CZT spectra reported in this paper.

\section{Summary}

We have reported parameters that describe the peak shapes in gamma spectra taken with cadmium zinc telluride detectors. We describe each peak as a sum of a Gaussian and an exponential tail, and report the variation of the parameters with gamma energy. Such a parameterization is very useful for the analysis of complex gamma spectra that one encounters in the field of safeguards technology such as the spectrum of uranium in the $100 \mathrm{keV}$ region.

* This work was performed under the auspices of the U.S. Department of Energy by the Lawrence Livermore National Laboratory under Contract No. W7405-Eng-48.

\section{References}

1) R. Arlt, K.-H. Czock and D.E. Rundquist, Nucl. Instr. Methods A322, (1992) 575 .

2) W.D. Ruhter and R. Gunnink, Nucl. Instr. Methods A353, (1994) 716 .

3) E. Raisin and J.F. Butler, IEEE Trans. Nucl. Sci, 35 (1988), 81.

4) F.P. Doty, J.F. Butler, J.F. Schetzina and K.A. Bowers, J. Vac. Sci. Technol. B10 (1992), 1418.

5) A.D. Lavietes, J.H. McQuaid, W.D. Ruhter and T.J. Paulus, Development of a Portable Ambient Temperature Radiometric Assaying Instrument, Lawrence Livermore National Laboratory Report, UCRL-J-117141 (1994); IEEE Trans. Nucl. Sci. 42 no. 4, 634 (1995); A.D. Lavietes, J.H. McQuaid, W.D. Ruhter, W.M. Buckley and T.J. Paulus, Proc. of the 37th Annual Meeting of the Inst. of Nucl. Materials Management, Naples, Florida (1996).

6) C. Johnson et al. Mater. Res. Soc. Symp. Proc., 302 (1993) 463.

7) M. Reichert and P. Siffert, Nucl. Instr. and Meth., A232 (1992) 529.

8) Y. Eisen and Y. Horowitz, Nucl. Instr. and Meth., A353 (1994) 60.

9) Y. Takenaka et al., Nucl. Instr. and Meth., A369 (1996) 637.

10) J.F. Briesmeister (ed.), ' MCNP - A General Monte Carlo Code for Neutron and Proton Transport', Version 4., Los Alamos National Laboratory (1994). 11) R. Gunnink, W.D. Ruhter and J.B. Niday, GRPANL: A suite of Computer Programs for Analyzing Complex Ge and Alpha-Particle Spectra, Lawrence Livermore National Laboratory Report, UCRL-53861, Vol. 1 (1988). 
12) D. Clark, W.M. Buckley and W.D. Ruhter, LLNL, Work in progress (1996).

13) D. Clark, private communication (1966)

14) M.G. Scannavini et al., Nucl. Instr. Meth. A353 (1994), 80.

\section{Figure captions:}

Fig. 1: $\quad$ Schematic of the electronic setup developed for the portable CZT system (ref. 3)

Fig. 2(a): A spectrum of the 122-keV gamma ray from ${ }^{57}$ Co taken with the CZT detector. (b) A Monte Carlo simulation of the spectrum produced by 122 $\mathrm{keV}$ gamma rays incident on the detector. Detector resolution and effects of carrier trapping are not included in the calculation.

Fig. 3. A spectrum of the $122-\mathrm{keV}$ gamma ray from 57 Co taken with the CZT detector. The components from the fitting procedure are shown.

Fig. 4: $\quad$ Energy dependence of the variance of the Gaussian component.

Fig. 5: (a): Energy dependence of the slope of the tail. The line through the data for detector. A (bottom) has the parameterization, Slope $=4.13 . \exp \left(-0.0281 \mathrm{E}_{\gamma}\right)+0.492 \exp \left(-0.00103 \mathrm{E}_{\gamma}\right)$, with $\mathrm{E}_{\gamma}$ expressed in $\mathrm{keV}$. This curve is scaled by a factor of 0.72 and plotted along with the data for detectors $B$ and $C$ on the top. (b) Energy dependence of the tail amplitude (given as a ratio to the Gaussian peak height). The line through detector A data has the parameterization,

Tail amplitude relative to peak $=1.583\left[1-\exp \left(0.00375 \mathrm{E}_{\gamma}\right)\right]+0.127$ with $E_{\gamma}$ expressed in $\mathrm{keV}$. This curve is scaled by a factor of 0.95 and plotted along with the data for detectors $B$ and $C$ on the top.

Fig. 6: Comparison of spectra taken with detectors from two different manufacturers.

Fig. 7: $\quad$ Comparison of the shape parameters for the $122 \mathrm{keV}$ peak in the spectra shown in Fig. 6. 


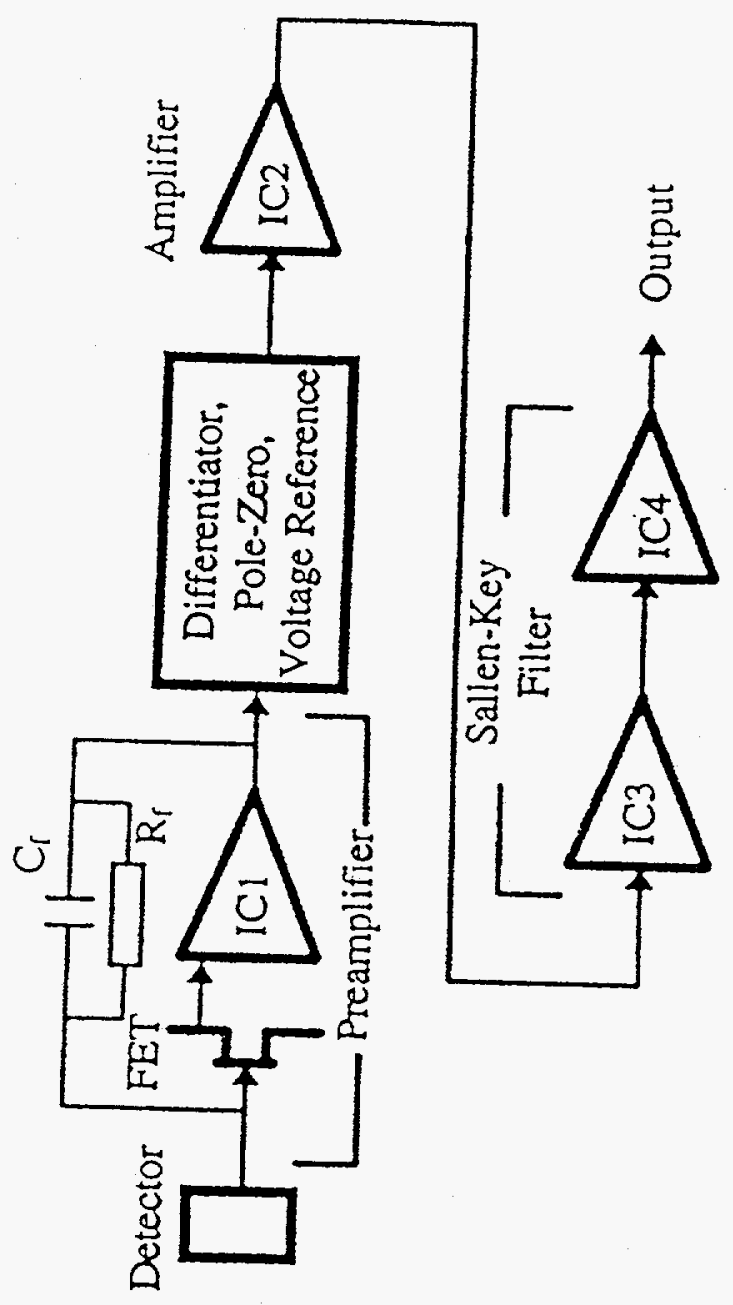




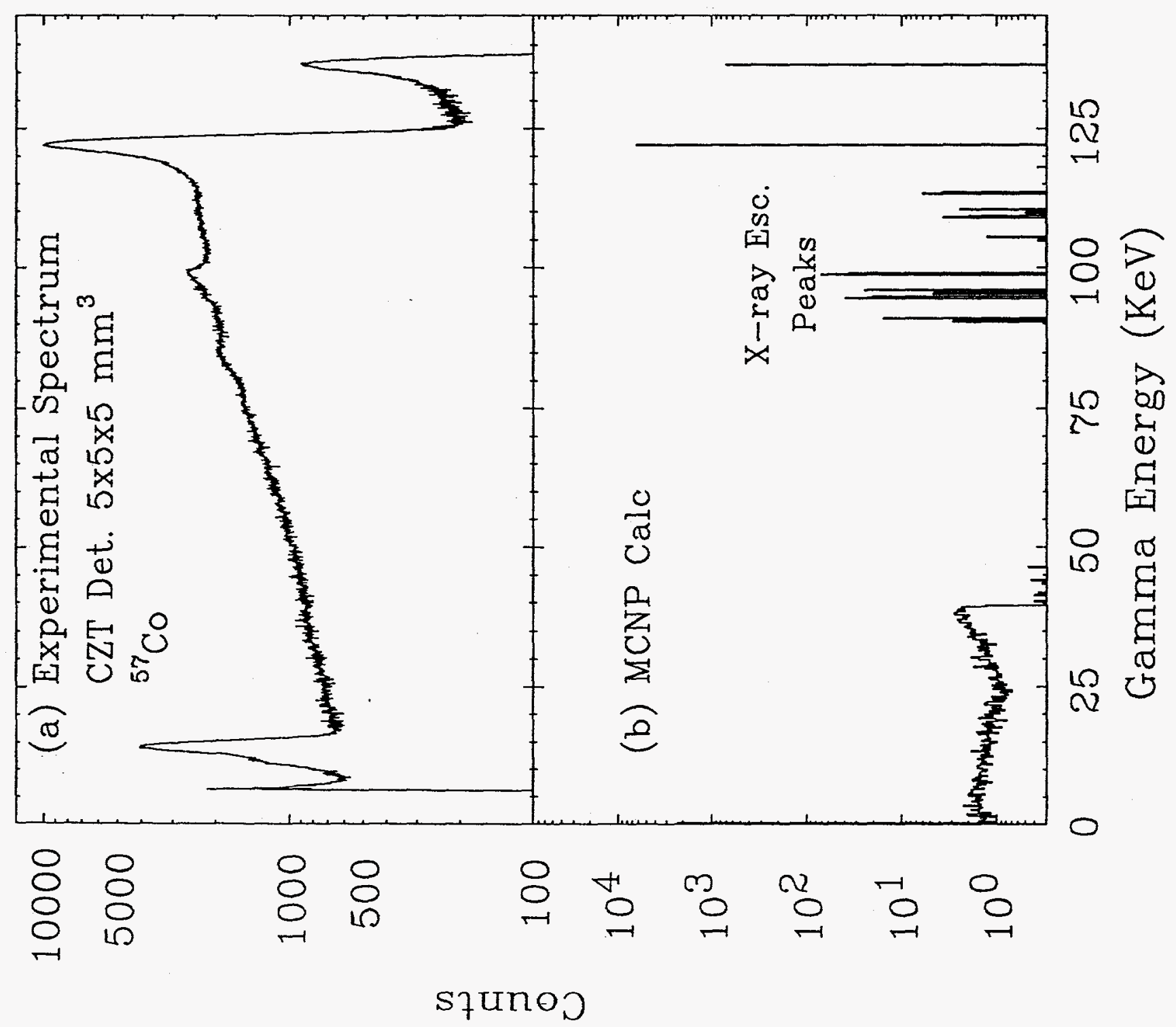




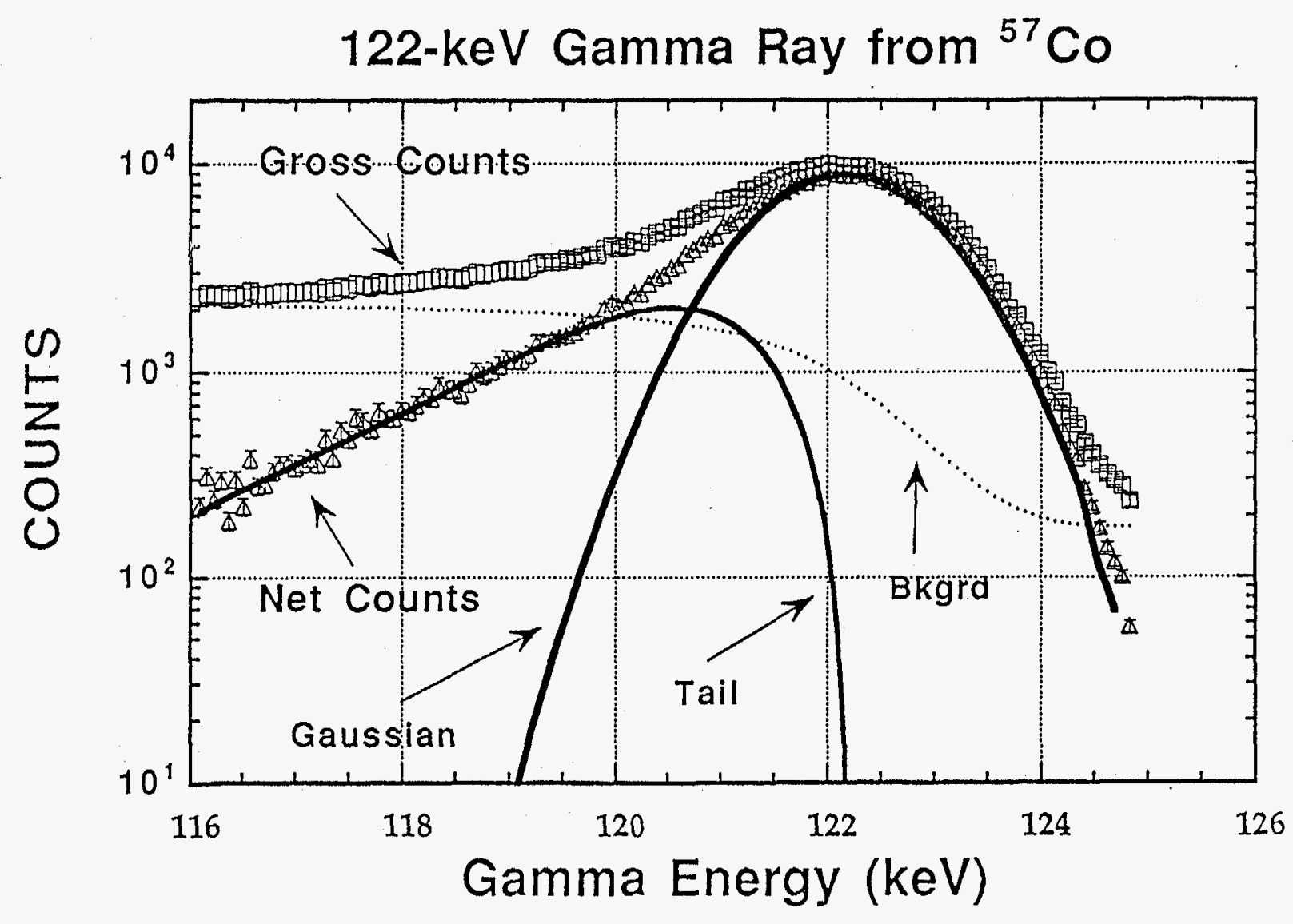




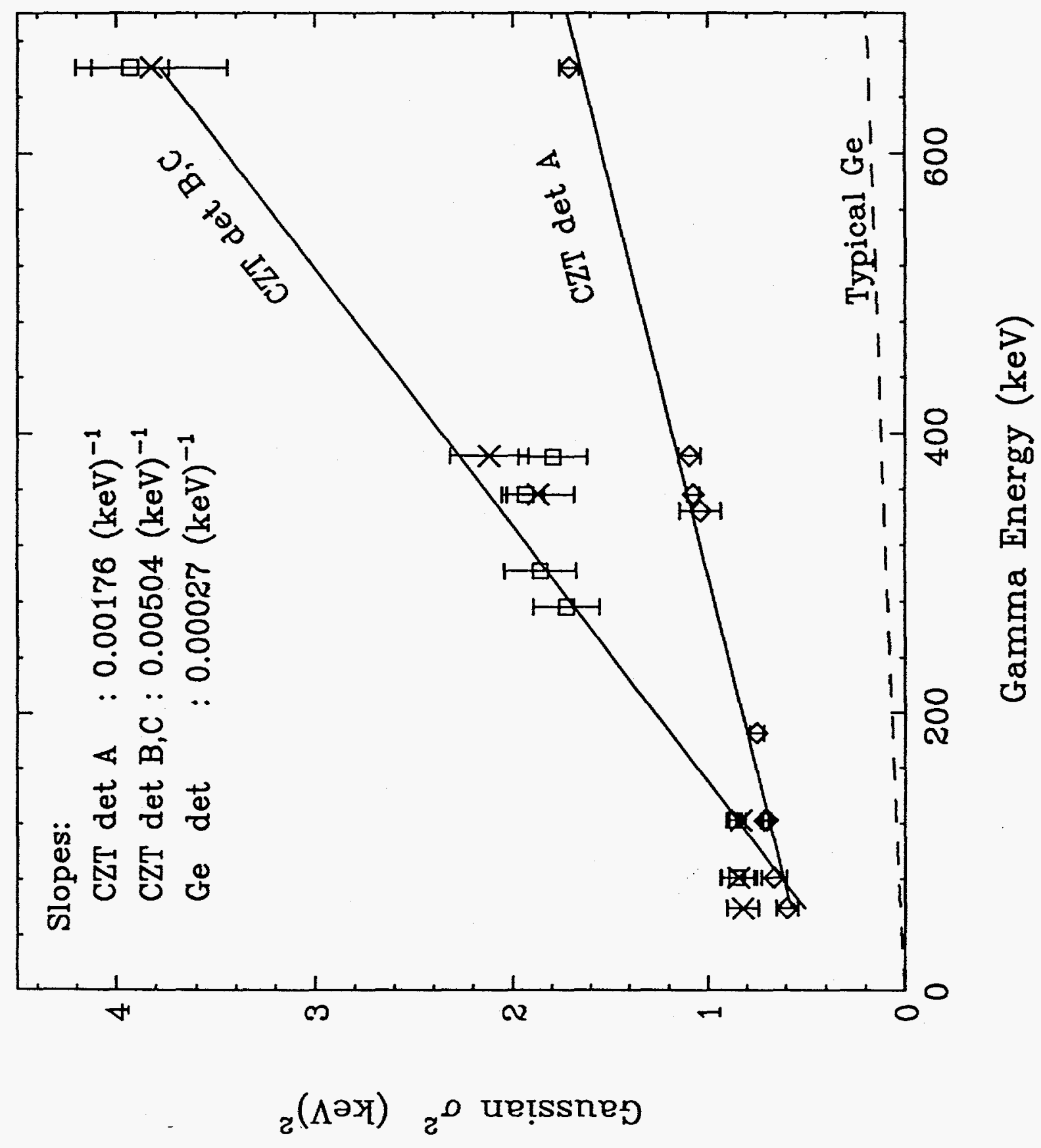




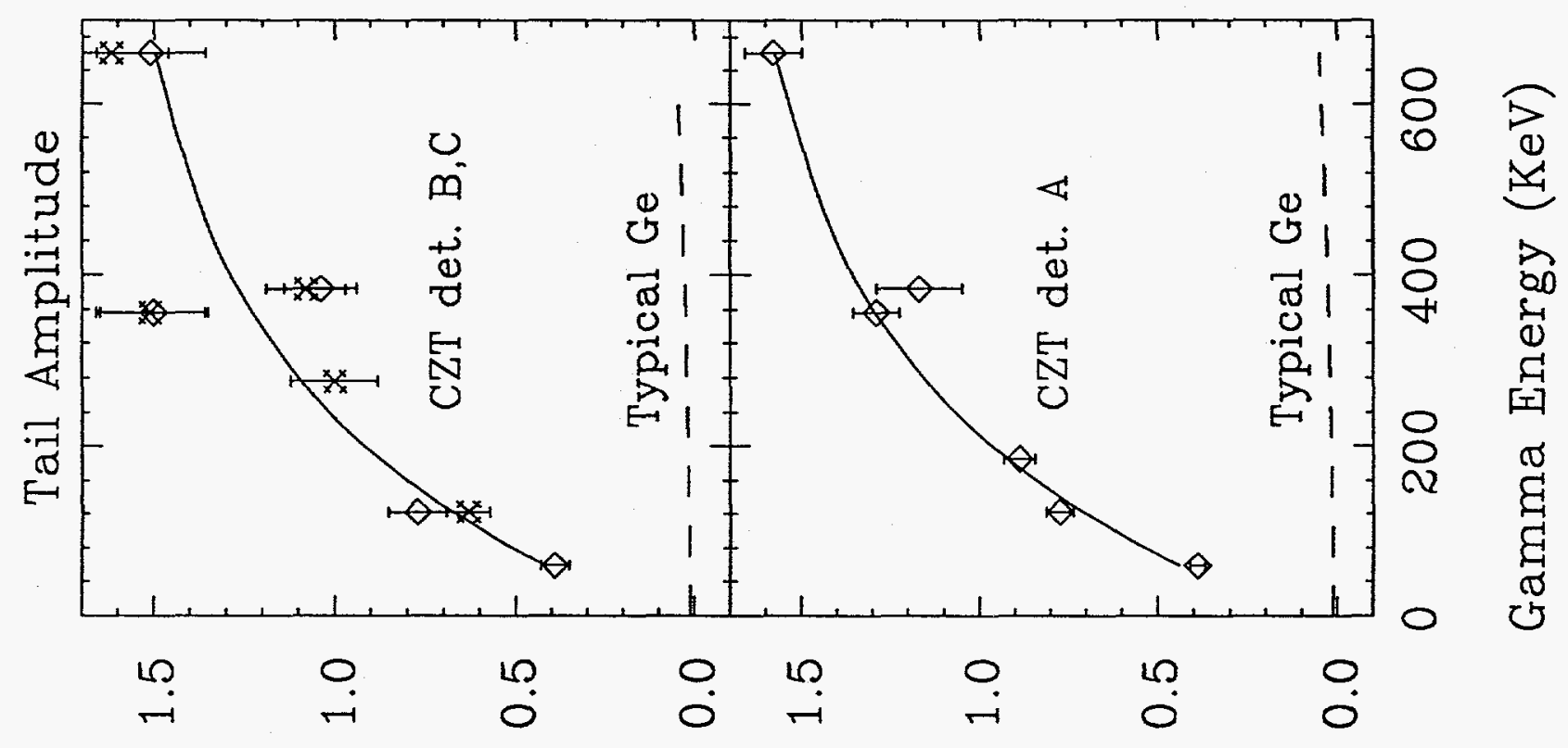

7H Yd 'ssneग/ [dur Ițe

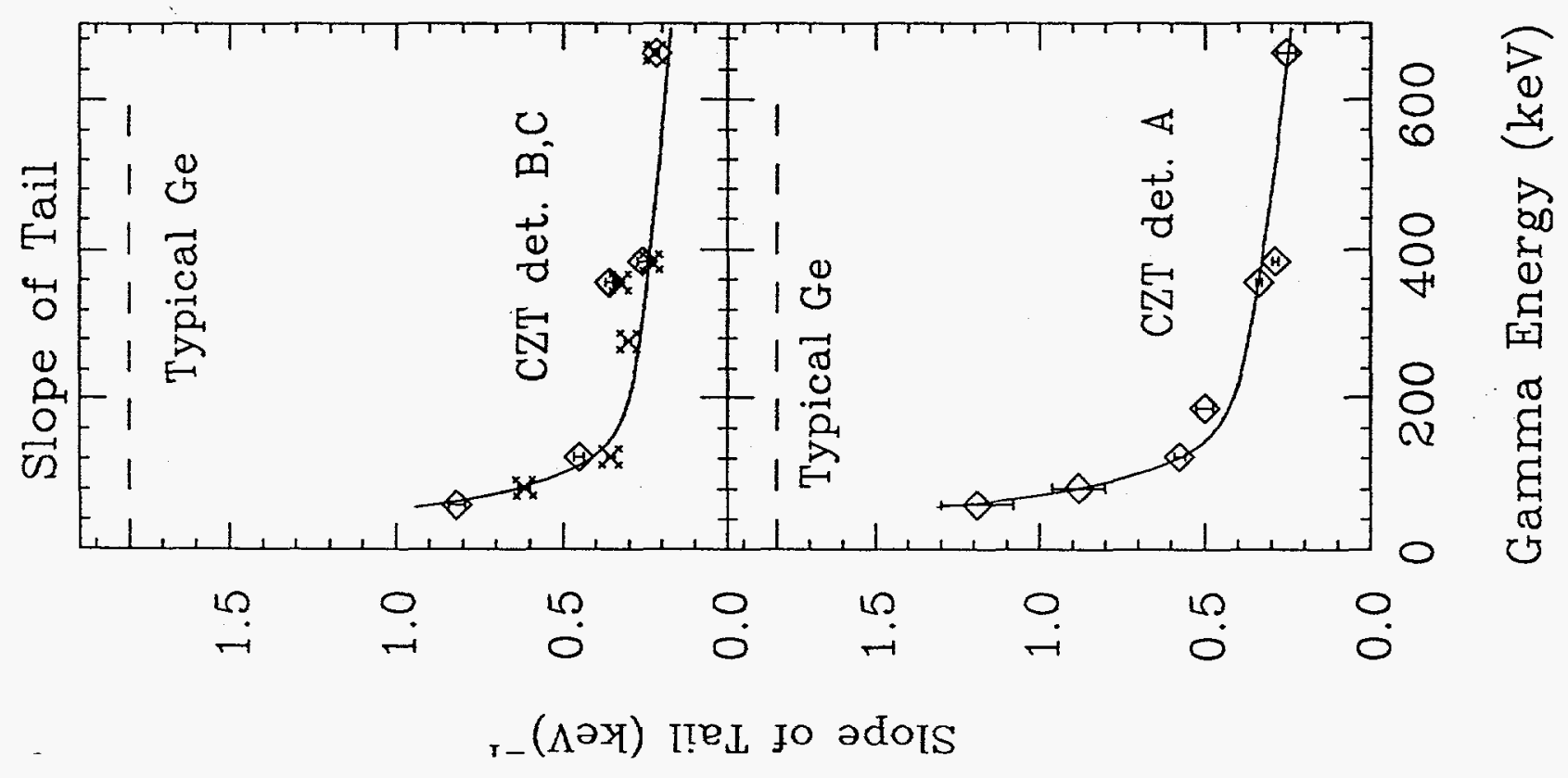




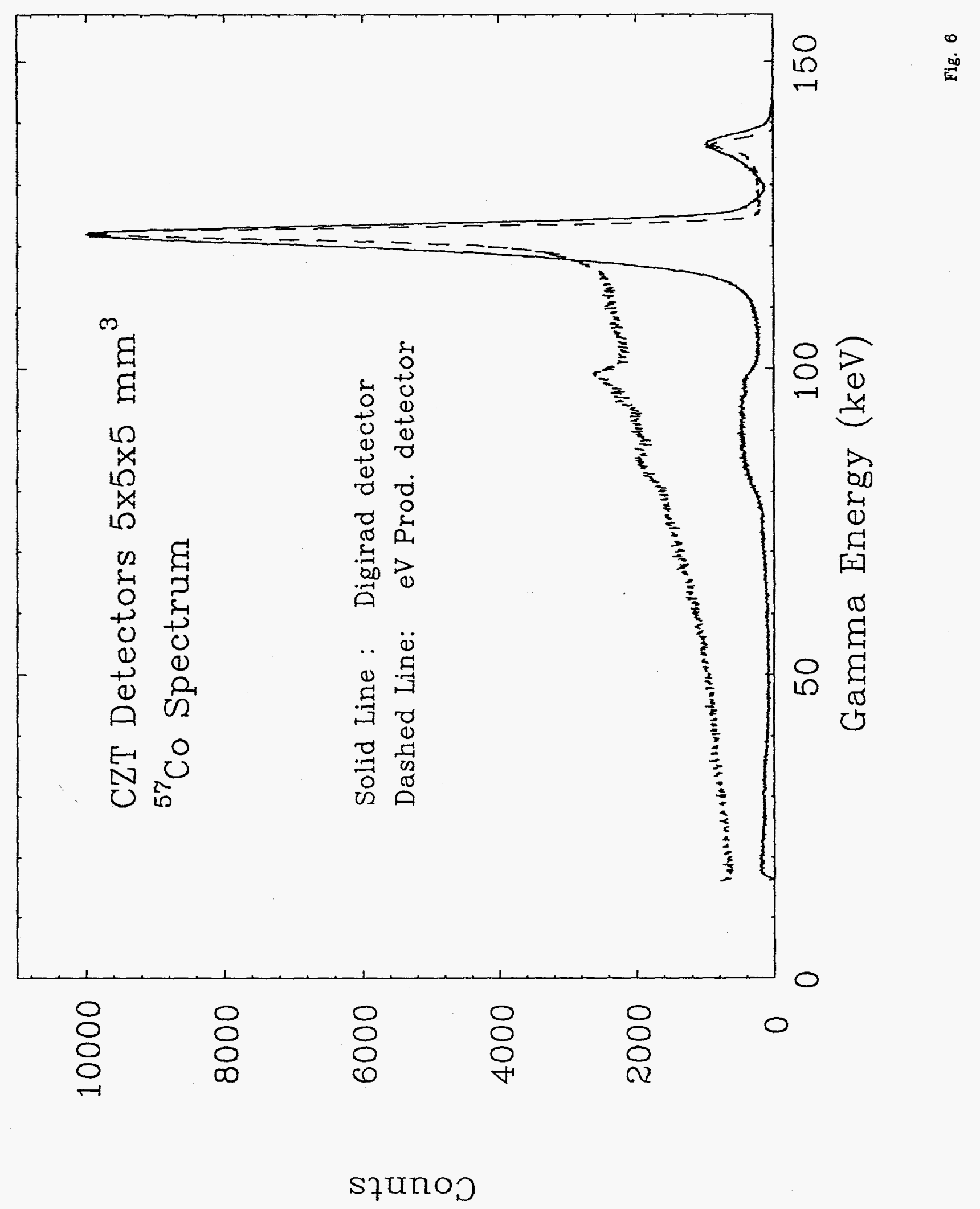



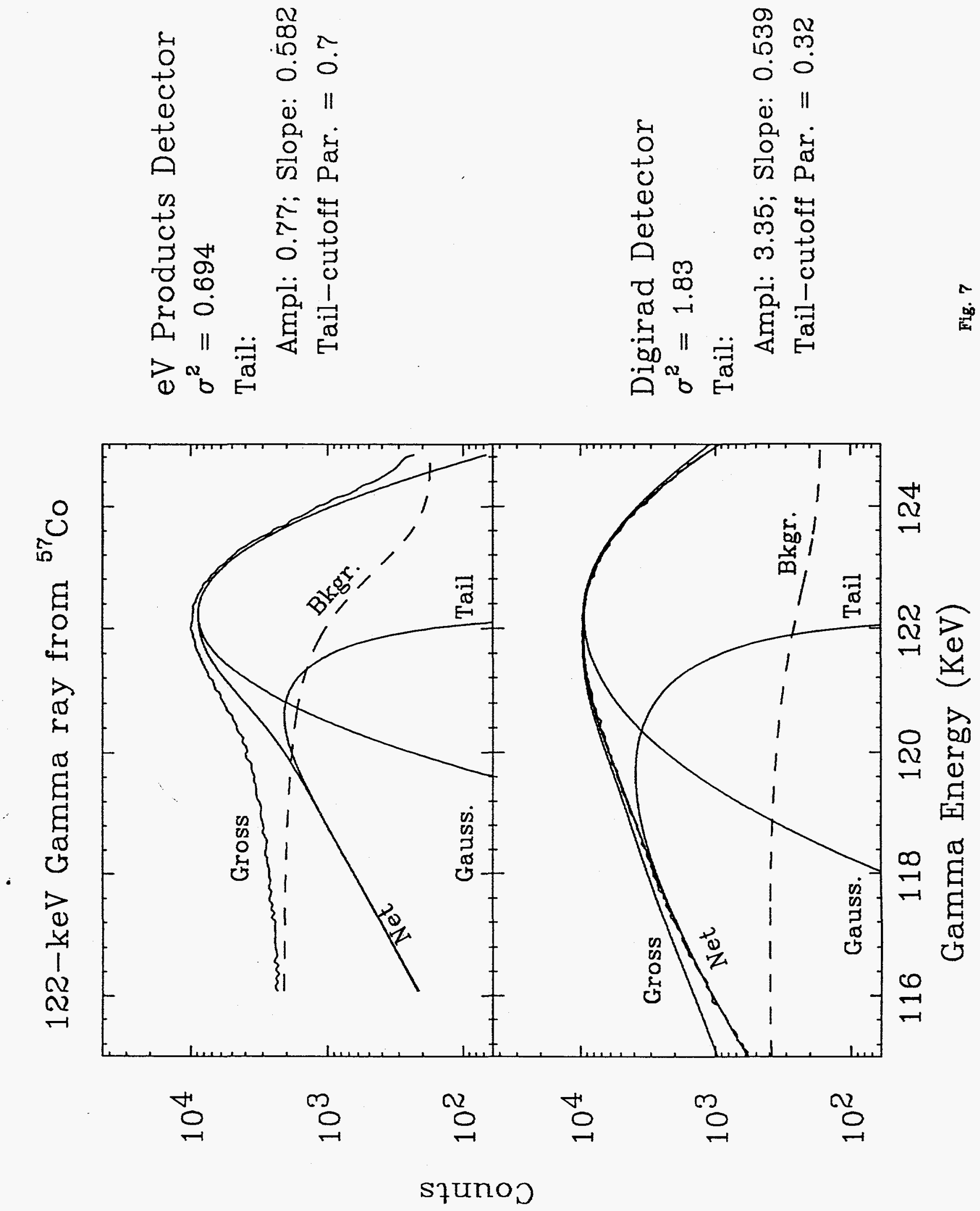